\title{
Generation time of the Alpha and Delta SARS-CoV-2 variants
}

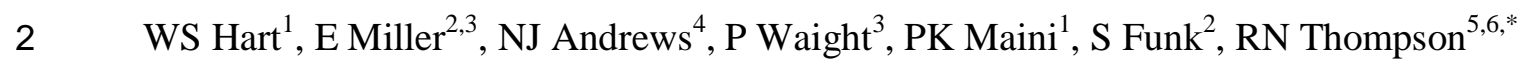

\section{Affiliations:}

$4 \quad{ }^{1}$ Mathematical Institute, University of Oxford, Oxford, OX2 6GG, UK

$5 \quad{ }^{2}$ Department of Infectious Disease Epidemiology, London School of Hygiene and Tropical

6 Medicine, London, WC1E 7HT, UK

$7 \quad{ }^{3}$ Immunisation and Countermeasures Division, UK Health Security Agency, London, NW9

$8 \quad 5 \mathrm{EQ}, \mathrm{UK}$

$9 \quad{ }^{4}$ Data and Analytical Sciences, UK Health Security Agency, London, NW9 5EQ, UK

$10{ }^{5}$ Mathematics Institute, University of Warwick, Coventry, CV4 7AL, UK

$11{ }^{6}$ Zeeman Institute for Systems Biology and Infectious Disease Epidemiology Research

12 (SBIDER), University of Warwick, Coventry, CV4 7AL, UK

$13 *$ Correspondence to: Robin.N.Thompson@warwick.ac.uk 
15 Background: In May 2021, the Delta SARS-CoV-2 variant became dominant in the UK.

16 This variant is associated with increased transmissibility compared to the Alpha variant that

17 was previously dominant. To understand ongoing transmission and interventions, a key

18 question is whether the Delta variant generation time (the time between infections in infector-

19 infectee pairs) is typically shorter-i.e., transmissions are happening more quickly-or whether

20 infected individuals simply generate more infections.

21 Methods: We analysed transmission data from a UK Health Security Agency household

22 study. By fitting a mathematical transmission model to the data, we estimated the generation

23 times for the Alpha and Delta variants.

24 Results: The mean intrinsic generation time (the generation time if there had been a constant 25 supply of susceptibles throughout infection) was shorter for the Delta variant (4.6 days, $95 \%$

$26 \mathrm{CrI} 4 \cdot 0-5 \cdot 4$ days) than the Alpha variant (5.5 days, 95\% CrI 4.6-6.4 days), although within

27 uncertainty ranges. However, there was a larger difference in the realised mean household

28 generation time between the Delta (3.2 days, 95\% CrI 2.4-4.2 days) and Alpha (4.5 days,

$2995 \%$ CrI 3.7-5.4 days) variants. This is because higher transmissibility led to faster

30 susceptible depletion in households, in addition to the reduced intrinsic generation time.

31 Conclusions: The Delta variant transmits more quickly than previously circulating variants.

32 This has implications for interventions such as contact tracing, testing and isolation, which

33 are less effective if the virus is transmitted quickly. Epidemiological models of interventions

34 should be updated to include the shorter generation time of the Delta variant. 
36 In mid-2021, the Delta SARS-CoV-2 variant became the dominant variant in the $\mathrm{UK}^{1}$ and

37 globally. ${ }^{2}$ This variant presents a higher risk of severe disease compared to previously

38 circulating variants, ${ }^{2,3}$ although vaccination still provides significant protection. ${ }^{4}$

39 Concerningly, the Delta variant led to an increase in the growth rate of COVID-19 cases in

40 the UK, swiftly outcompeting other SARS-CoV-2 variants. ${ }^{1,5}$ This was attributed to the

41 increased transmissibility of the Delta variant, with an epidemiological study ${ }^{5}$ suggesting that

42 it is $43-68 \%$ more transmissible than the Alpha variant that was identified in the UK in late

$432020 .^{6}$

44 Transmission of a SARS-CoV-2 variant can be characterised in two complementary ways:

45 speed and strength. ${ }^{7,8}$ Speed refers to how quickly the variant grows at the population level,

46 which is measured by the exponential growth rate and can be inferred from disease incidence

47 data for that variant. ${ }^{8}$ However, understanding transmission of a novel variant also requires its

48 strength to be estimated ${ }^{8}$ - this is the variant's transmissibility, which is typically measured

49 by the time-dependent reproduction number, or " $\mathrm{R}$ number" (the number of people that each

50 infected person is expected to infect). The generation time (the time between infection events

51 in infector-infectee pairs) determines the relationship between the speed and strength of a

52 variant, ${ }^{8-11}$ and is a crucial input to many models used to estimate the reproduction number

53 from case notification data. ${ }^{12,13}$ In principle, an increase in the growth rate of COVID-19

54 cases as observed for the Delta variant can be attributed to either an increase in

55 transmissibility or a shortening of the generation time, or both of these factors. ${ }^{8,14}$

56 Previous studies have estimated the generation time for SARS-CoV-2 (for example ${ }^{15-18}$ ), with

57 most of these estimates using data collected during the very early months of the COVID-19

58 pandemic. A household study conducted by Public Health England (PHE) between February 
59 and November 2020 in the UK indicated that the generation time became shorter in the 60 autumn of 2020 compared to earlier months. ${ }^{18}$ Since the SARS-CoV-2 generation time is

61 changing, up-to-date generation time estimates are crucial to inform estimates of the

62 reproduction number and to understand the relative transmissibility of different variants.

63 However, the Alpha variant was responsible for infections in only two households in that

64 study, while the Delta variant had not yet emerged by the end of the study. ${ }^{18}$ The effect of

65 different variants on the SARS-CoV-2 generation time has therefore not been properly

66 assessed.

67 Here, we report analyses of data from an ongoing household study conducted by the UK

68 Health Security Agency (UKHSA; formerly PHE). As the study began in February 2021,

69 there was an opportunity to estimate the generation time for both the Alpha and Delta

70 variants and to analyse the transition from the Alpha variant to the Delta variant being

71 dominant. We estimated two quantities for each variant. First, we estimated the intrinsic

72 generation time (the generation time if the supply of susceptible individuals remains constant

73 throughout infection). This quantity is widely applicable, since its distribution describes the

74 relative transmission risk posed by an infected host at each time since infection,

75 independently of the household structure. Second, we estimated the household generation

76 time (the realised generation time in the study households). The mean household generation

77 time is always shorter than the mean intrinsic generation time. This is because the household

78 generation time reflects the fact that, in small household populations, infected individuals run

79 out of susceptible individuals to infect. ${ }^{9,19}$ Most realised transmissions therefore happen more

80 quickly within households than the intrinsic generation time distribution suggests.

81 In addition to estimating the household generation time for the Alpha and Delta variants, we 
83 different times during the study period. We found that the variant responsible for the

84 infections within households had a larger effect on the household generation time than any of

85 these other factors. This indicates that the increased growth rate of COVID-19 cases due to

86 the Delta variant can be attributed to both an increase in transmissibility and a shortening of

87 the generation time.

\section{$\underline{\text { Methods }}$}

$89 \underline{\text { Data }}$

90 We analysed household transmission data from the UKHSA HOCO2 study (Supplementary

91 Data). This study began in February 2021 and is ongoing, and data were available from 227

92 households consisting of 557 participants at the time of our analyses (September 2021).

93 Households were recruited to the study after an index case returned a positive PCR test, and

94 (in most cases) three further PCR tests were subsequently carried out on each participating

95 household member to determine whether they became infected. The following data were also

96 collected from each participating household member:

97 - The date of any previous positive test result, where available.

98 - Symptom onset dates (for individuals who developed symptoms).

99 - The number of vaccine doses received, the vaccine type, and the date(s) of vaccination.

100 We assumed individuals were infected during the household transmission cluster if they

101 returned a positive PCR test (including tests taken up to 28 days before the household was

102 recruited to the study) and/or developed symptoms. Otherwise, we assumed individuals

103 remained uninfected if they returned only negative tests. Household members who did not

104 participate in the study, or withdrew before either taking a test or developing symptoms, were

105 excluded from our analyses (but were included in the household size). 
106 Genomic sequencing was carried out in $60 \%$ of study households. Where sequencing data

107 were not available, we assumed that the Alpha variant was responsible for infections in

108 households that were recruited to the study before May 2021, and the Delta variant for

109 infections in households recruited after May 2021 (data from three households recruited

110 during May 2021 in which sequencing was not carried out were excluded from our

111 analyses). ${ }^{1}$ Overall, the Alpha and Delta variants were determined to be responsible for

112 infections in 131 and 96 households, respectively.

113 Mathematical modelling

114 We provide an overview of the model and the parameter inference approach here. Full details

115 are given in the Supplementary Material.

116 We estimated the generation time from the household transmission data using a mechanistic

117 approach motivated by compartmental modelling. ${ }^{17,18}$ In the model, each infection is divided

118 into three successive, independently distributed, stages: latent, presymptomatic infectious and

119 symptomatic infectious. Unlike commonly used approaches for estimating the SARS-CoV-2

120 generation time, ${ }^{15,16}$ this approach explicitly links the individual infectiousness profile of an

121 infected host to exactly when they develop symptoms.

122 Data augmentation MCMC was used to fit the transmission model to the household data. ${ }^{18,20}$

123 We made the following assumptions when fitting the model to data:

124 - An incubation period distribution with mean 5.8 days and standard deviation 3.1

125 days. $^{21}$

126 Entirely asymptomatic infected hosts are $35 \%$ as infectious as those who develop

127 symptoms. ${ }^{22}$ 
- Reduced susceptibility of vaccinated individuals compared to unvaccinated individuals (a ‘leaky’ vaccine was assumed), using estimates of protection against infection obtained by Pouwels and colleagues (Table S1). ${ }^{23}$

- All household transmission clusters in the study originated with a single primary case, with no further infections introduced into the household from outside.

134 the parameter fitting procedure:

135 - The mean latent period (the time from being infected to becoming infectious), as a

136 proportion of the mean incubation period.

- The mean symptomatic infectious period.

- The relative infectiousness of presymptomatic infectious hosts compared to those with symptoms.

- The overall transmissibility parameter, $\beta_{0}$, which describes the expected number of household transmissions generated by a single infector (who develops symptoms) in a large, otherwise entirely susceptible and unvaccinated, household.

143 Posterior distributions of fitted model parameters are shown in Figure S1.

144 The intrinsic generation time distribution was calculated for each variant from the assumed

145 and estimated values of model parameters. Realised generation times within the study

146 households were also estimated at each step of the MCMC procedure. This enabled us to

147 compare household generation times based on variant, vaccination status, date and age.

\section{$\underline{\text { Results }}$}

149 In Figure 1A, we show posterior estimates of the mean intrinsic generation time (which

150 involves an assumption that infected individuals have contacts with susceptible hosts at a 
A.

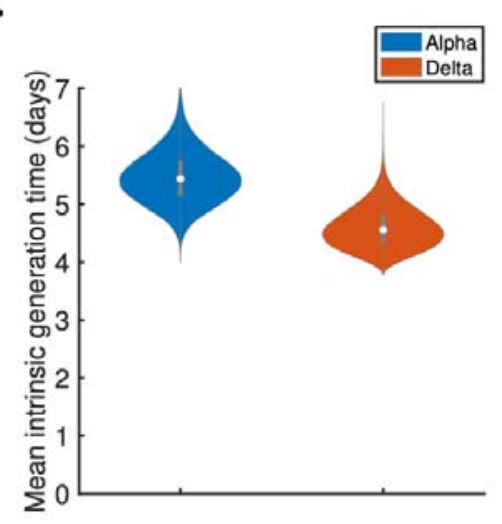

C.
B.

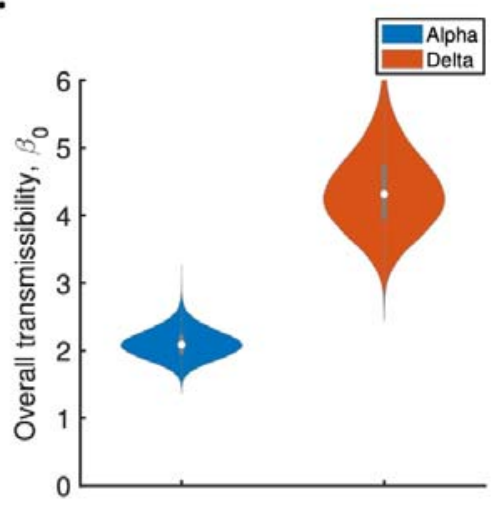

D.

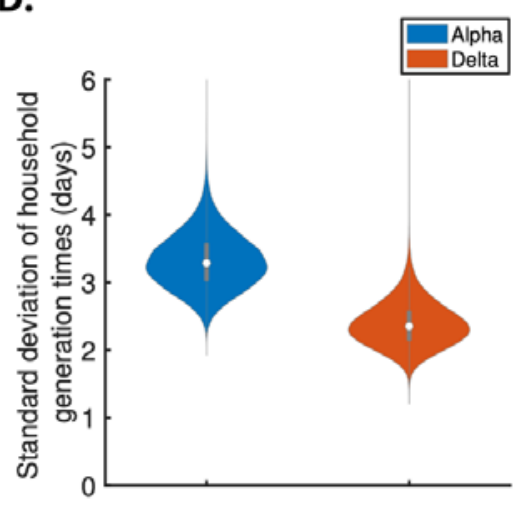

Figure 1. The effect of variant on the intrinsic generation time and the household generation time. Violin plots indicate posterior estimates for the Alpha (blue) and Delta (red) variants of: A. The mean intrinsic generation time (i.e., the mean generation time if the supply of susceptibles remains constant throughout infection); B. The overall transmissibility parameter, $\beta_{0}$ (i.e., the expected number of household transmissions generated by a single infected individual introduced into a large, entirely susceptible, household); C. The mean household generation time (i.e., the mean generation time accounting for susceptible depletion in the 
medRxiv preprint doi: https://doi.org/10.1101/2021.10.21.21265216; this version posted October 23, 2021. The copyright holder for this preprint (which was not certified by peer review) is the author/funder, who has granted medRxiv a license to display the preprint in perpetuity.

It is made available under a CC-BY-NC 4.0 International license.

households); D. The standard deviation of household generation times. Posterior means and 95\% credible intervals for these quantities are given in Table S2 and Table S3 in the Supplementary Material.

Estimates of the mean household generation time for the two variants (Figure 1C) are shorter than the corresponding intrinsic generation time estimates (Figure 1A), since the household generation time accounts for susceptible depletion within households (which means that potential transmissions with longer generation times are less likely to occur). We found a shorter mean household generation time for the Delta variant $(3 \cdot 2$ days, $95 \%$ CrI 2.4-4.2 days) than for the Alpha variant ( 4.5 days, $95 \%$ CrI 3.7-5.4 days). This is due to the higher transmissibility of the Delta variant (Figure 1B) leading to faster susceptible depletion, in addition to the smaller reduction in the intrinsic generation time shown in Figure 1A. We also estimated the standard deviation of generation times within households to be smaller for the Delta variant than for the Alpha variant (Figure 1D). In Figure S2 of the Supplementary Material, we combined the estimates obtained in each step of the data augmentation MCMC parameter fitting procedure (which are summarised in Figure 1) to obtain an estimate of the entire household generation time distribution. This also indicates that household transmissions with the Delta variant typically occur earlier in infection (Figure S2C).

We note that the estimates in Figure 1B cannot be compared directly with previous estimates of the transmission advantage of the Delta variant (e.g., an estimate of 43-68\% obtained using incidence data ${ }^{5}$ ), since the value of $\beta_{0}$ reflects the instrinsic transmissibility of the Alpha and Delta variants rather than realised transmission. The intrinsic transmission advantage of the Delta variant will only be realised if sufficiently many susceptibles are available to each infector.

We also explored the effects of vaccination (Figure 2A-C), age (Figure 2D-E) and month of recruitment to the household study (Figure $2 \mathrm{~F}$ ) on household generation times. We estimated 
medRxiv preprint doi: https://doi.org/10.1101/2021.10.21.21265216; this version posted October 23, 2021. The copyright holder for this preprint (which was not certified by peer review) is the author/funder, who has granted medRxiv a license to display the preprint in perpetuity.

It is made available under a CC-BY-NC 4.0 International license .

189

190

191

192

A.

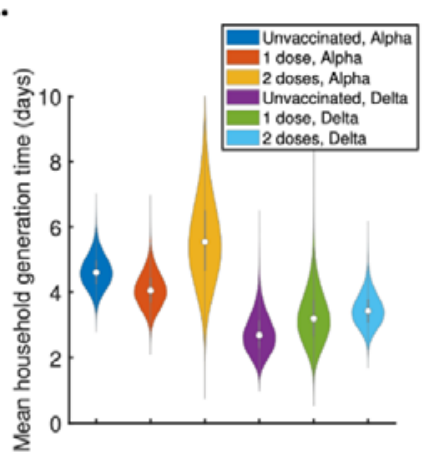

D.

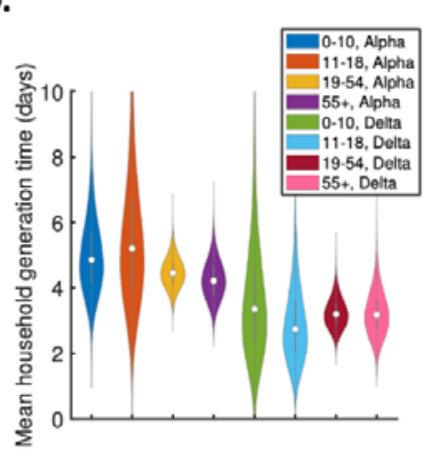

B.

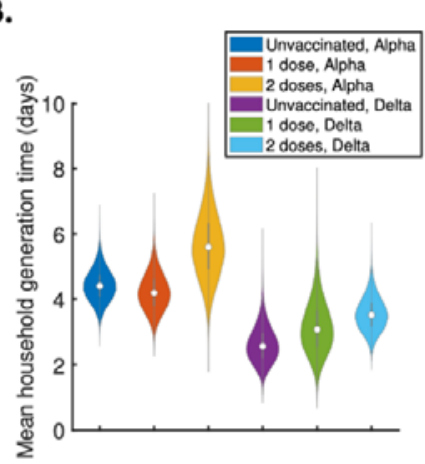

E.

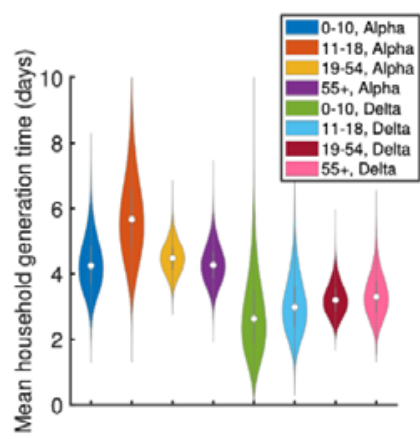

C.

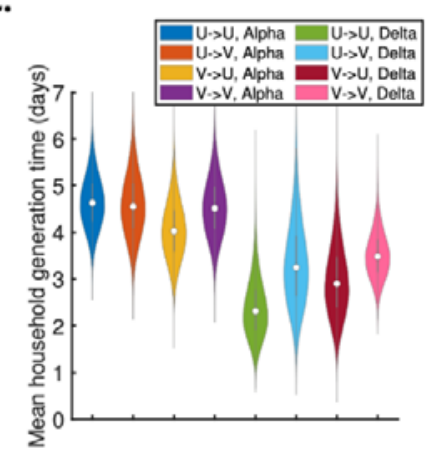

F.

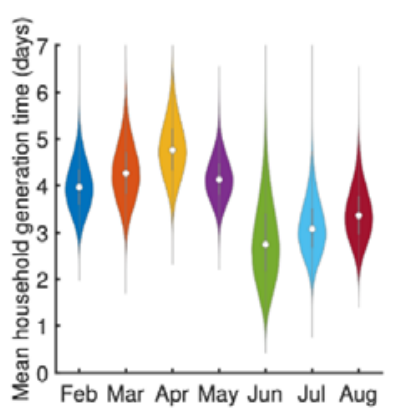

Figure 2. The effect of different factors on household generation times. Violin plots indicate posterior

estimates of the mean household generation time for infectors, infectees and households with different 
medRxiv preprint doi: https://doi.org/10.1101/2021.10.21.21265216; this version posted October 23, 2021. The copyright holder for this preprint (which was not certified by peer review) is the author/funder, who has granted medRxiv a license to display the preprint in perpetuity.

It is made available under a CC-BY-NC 4.0 International license .

\section{Discussion}

206 Novel SARS-CoV-2 variants possess different transmission characteristics to the virus that 207 originally emerged in Wuhan, China., ${ }^{5,14}$ A key transmission characteristic is the generation 208 time, which measures the speed of transmission between individuals. ${ }^{17,19,24}$ To estimate the 209 generation time, it is necessary to analyse datasets in which it is possible to determine or

210 estimate who-infected-whom. ${ }^{17,19,24}$ Household studies provide an opportunity to conduct such

211 analyses. ${ }^{18,20}$ Here, we have analysed data from the HOCO2 household study conducted by

212 the UKHSA, and compared the generation time for the Alpha and Delta variants (which were

213 the dominant variants in England at the beginning and end of the time period considered ${ }^{1}$ ).

214 We first estimated the intrinsic generation time, which describes the generation time under

215 the assumption that there is a steady supply of susceptible individuals available to infect (i.e.,

216 susceptible depletion in households is not accounted for). The mean intrinsic generation time

217 was found to be slightly lower for the Delta variant than for the Alpha variant (Figure 1A),

218 but there was a substantial overlap in the credible intervals between the two variants.

219 However, the data indicated that the Delta variant is much more transmissible in households

220 than the Alpha variant (Figure 1B). The impact of this increased transmissibility is a larger

221 difference in realised generation times within households between the two variants (Figure

222 1C) than would be expected from the difference in the intrinsic generation time alone. This is

223 because increased transmissibility leads to susceptible individuals in the household being

224 infected quickly, thereby being unavailable for infection later (thus shortening the average

225 period between realised transmissions). The effect of variant on the household generation

226 time was more substantial than the effects of other factors, including age and vaccination 227 status (Figure 2). 
medRxiv preprint doi: https://doi.org/10.1101/2021.10.21.21265216; this version posted October 23, 2021. The copyright holder for this preprint (which was not certified by peer review) is the author/funder, who has granted medRxiv a license to display the preprint in perpetuity.

It is made available under a CC-BY-NC 4.0 International license.

228 Our study is the first to compare the generation time for different SARS-CoV-2 variants

229 directly, and we are not aware of any other generation time estimate for the Alpha variant. A

230 generation time estimate for the Delta variant based on 55 transmission pairs was recently

231 stated in China CDC Weekly, ${ }^{25}$ although the methods underlying that estimate were not

232 presented. Nonetheless, the mean generation time estimate of 2.9 days in that $s^{2} u d y^{25}$ lies

233 within the credible interval of our estimate of the mean household generation time for the

234 Delta variant (3.2 days, $95 \%$ CrI 2.4-4.2 days) and is shorter than the lower credible limit of

235 our estimate for the Alpha variant (4.5 days, 95\% CrI 3.7-5.4 days). This supports our finding

236 that the Delta variant is transmitted more quickly than the Alpha variant.

237 Other studies have estimated quantities related to the generation time for the Alpha and Delta

238 variants, including the serial interval ${ }^{26-28}$ and the viral load trajectories of infected hosts. ${ }^{29-31}$

239 However, while the serial interval (the interval between symptom onset times in infector-

240 infectee pairs) is sometimes used as a proxy for the generation time (the interval between

241 infection times), these two quantities may not follow the same distribution ${ }^{24}$ - for example,

242 the generation time always takes positive values, whereas the serial interval can be negative

243 for pathogens such as SARS-CoV-2 for which presymptomatic transmission can occur - and

244 may not even have the same mean value. ${ }^{18}$ Similarly, although it may be possible to infer how

245 infectiousness varies during infection based on viral load data, ${ }^{32}$ the timing of realised

246 transmissions also depends on other factors as well as intrinsic infectiousness, such as

247 behaviour (for example, individuals may be less likely to transmit after developing symptoms

248 and isolating) and the availability of susceptibles. Our approach explicitly accounts for

249 changes in the transmission risk following symptom onset, while our estimates of the

250 household generation time account for depletion of susceptibles within households. 
251 Estimates of the generation time underlie a range of epidemiological analyses, including

252 estimation of the time-varying reproduction number (or " $\mathrm{R}$ number") ${ }^{12,13}$ in different regions

253 or countries and the relative transmissibility of different variants ${ }^{5,14}$. Such analyses often

254 neglect temporal changes in the generation time, but our results indicate that the generation

255 time is shorter for the Delta variant than for the Alpha variant. When estimating reproduction

256 numbers, overestimation of the mean generation time generally leads to reproduction number

257 estimates that are further from one (the threshold for an outbreak being under control)

258 compared to the true value. ${ }^{10}$ When analysing the transmissibility advantage of the Delta

259 variant over the Alpha variant, neglecting a shorter generation time for the Delta variant

260 would lead to its transmissibility advantage being overestimated. ${ }^{8}$ We contend that the

261 observed growth rate advantage of the Delta variant is likely due to a combination of both

262 increased transmissibility and a shorter generation time.

263 Since susceptible depletion during infection may be a less important determinant of the

264 timing of transmissions occurring outside households than within households, we expect the

265 overall generation time distribution (accounting for transmissions across all settings) to lie

266 somewhere between our "household" and "intrinsic" estimates. This suggests that inferring

267 the transmissibility advantage of the Delta variant over the Alpha variant from incidence data

268 using our household generation time estimates (which show a greater difference between

269 variants) would give a lower bound for the transmissibility advantage (since the observed

270 increased growth rate of the Delta variant is then explained in part by a substantial generation

271 time reduction). In contrast, we expect that inferring the transmissibility advantage of the

272 Delta variant over the Alpha variant using the intrinsic generation time estimates would give

273 an upper bound. 
medRxiv preprint doi: https://doi.org/10.1101/2021.10.21.21265216; this version posted October 23, 2021. The copyright holder for this preprint (which was not certified by peer review) is the author/funder, who has granted medRxiv a license to display the preprint in perpetuity.

It is made available under a CC-BY-NC 4.0 International license .

274 In summary, our analyses suggests that the Delta variant transmits more quickly than the

275 previously circulating Alpha variant. This has implications for interventions, since public

276 health measures such as contact tracing are less effective if transmission occurs quickly. It is

277 essential for public health that epidemiological models are updated to reflect the generation

278 time of the variants driving transmission, and that the generation time is assessed in future as

279 the characteristics of SARS-CoV-2 transmission continue to change.

$280 \underline{\text { References }}$

281

282

283

284

285

286

287

288

289

290

291

292

293

294

295

296

297

298

299

300

301

302

303

304

305
1 Public Health England. SARS-CoV-2 variants of concern and variants under investigation in England (Technical briefing 23). 2021.

2 World Health Organization. Weekly epidemiological update on COVID-19 (Edition 58). 2021.

3 Twohig KA, Nyberg T, Zaidi A, et al. Hospital admission and emergency care attendance risk for SARS-CoV-2 delta (B.1.617.2) compared with alpha (B.1.1.7) variants of concern: a cohort study. Lancet Infect Dis 2021.

4 Lopez Bernal J, Andrews N, Gower C, et al. Effectiveness of Covid-19 vaccines against the B.1.617.2 (Delta) variant. N Engl J Med 2021; 385: 585-94.

5 Campbell F, Archer B, Laurenson-Schafer H, et al. Increased transmissibility and global spread of SARS-CoV-2 variants of concern as at June 2021. Eurosurveillance 2021; 26: 2100509.

6 Public Health England. SARS-CoV-2 variants of concern and variants under investigation in England (Technical briefing 7). 2021.

7 Dushoff J, Park SW. Speed and strength of an epidemic intervention. Proc R Soc B Biol Sci 2021; 288: 20201556.

8 Park SW, Bolker BM, Funk S, et al. Roles of generation-interval distributions in shaping relative epidemic strength, speed, and control of new SARS-CoV-2 variants. medRxiv 2021; : 2021.05.03.21256545.

9 Fraser C. Estimating Individual and Household Reproduction Numbers in an Emerging Epidemic. PLOS ONE 2007; 2: e758.

10 Wallinga J, Lipsitch M. How generation intervals shape the relationship between growth rates and reproductive numbers. Proc R Soc B Biol Sci 2007; 274: 599-604.

11 Parag KV, Thompson RN, Donnelly CA. Are epidemic growth rates more informative than reproduction numbers? Royal Statistical Society, 2021.

12 Gostic KM, McGough L, Baskerville EB, et al. Practical considerations for measuring the effective reproductive number, Rt. PLOS Comput Biol 2020; 16: e1008409. 
medRxiv preprint doi: https://doi.org/10.1101/2021.10.21.21265216; this version posted October 23, 2021. The copyright holder for this preprint (which was not certified by peer review) is the author/funder, who has granted medRxiv a license to display the preprint in perpetuity.

It is made available under a CC-BY-NC 4.0 International license .

306

307

308

309

310

311

312

313

314

315

316

317

318

319

320

321

322

323

324

325

326

327

328

329

330

331

332

333

334

335

336

337

338

339

340

341

342

343
13 Abbott S, Hellewell J, Thompson RN, et al. Estimating the time-varying reproduction number of SARS-CoV-2 using national and subnational case counts. Wellcome Open Res 2020; 5: 112.

14 Davies NG, Abbott S, Barnard RC, et al. Estimated transmissibility and impact of SARS-CoV-2 lineage B.1.1.7 in England. Science 2021; : eabg3055.

15 Ferretti L, Wymant C, Kendall M, et al. Quantifying SARS-CoV-2 transmission suggests epidemic control with digital contact tracing. Science 2020; 368: eabb6936.

16 Ganyani T, Kremer C, Chen D, et al. Estimating the generation interval for coronavirus disease (COVID-19) based on symptom onset data, March 2020. Eurosurveillance 2020; 25: 2000257.

17 Hart WS, Maini PK, Thompson RN. High infectiousness immediately before COVID-19 symptom onset highlights the importance of continued contact tracing. eLife 2021; 10: e65534.

18 Hart WS, Abbott S, Endo A, et al. Inference of SARS-CoV-2 generation times using UK household data. medRxiv 2021; : 2021.05.27.21257936.

19 Park SW, Champredon D, Dushoff J. Inferring generation-interval distributions from contacttracing data. J R Soc Interface 2020; 17: 20190719.

20 Ferguson NM, Cummings DAT, Cauchemez S, et al. Strategies for containing an emerging influenza pandemic in Southeast Asia. Nature 2005; 437: 209-14.

21 McAloon C, Collins Á, Hunt K, et al. Incubation period of COVID-19: a rapid systematic review and meta-analysis of observational research. BMJ Open 2020; 10: e039652.

22 Buitrago-Garcia D, Egli-Gany D, Counotte MJ, et al. Occurrence and transmission potential of asymptomatic and presymptomatic SARS-CoV-2 infections: A living systematic review and metaanalysis. PLOS Med 2020; 17: e1003346.

23 Pouwels KB, Pritchard E, Matthews PC, et al. Impact of Delta on viral burden and vaccine effectiveness against new SARS-CoV-2 infections in the UK. medRxiv 2021; : 2021.08.18.21262237.

24 Lehtinen S, Ashcroft P, Bonhoeffer S. On the relationship between serial interval, infectiousness profile and generation time. J R Soc Interface 2021; 18: 20200756.

25 Zhang M, Xiao J, Deng A, et al. Transmission dynamics of an outbreak of the COVID-19 Delta variant B.1.617.2 - Guangdong Province, China, May-June 2021. China CDC Wkly 2021; 3: $584-6$.

26 Pung R, Mak TM, Kucharski AJ, Lee VJ. Serial intervals in SARS-CoV-2 B.1.617.2 variant cases. The Lancet 2021; 398: 837-8.

27 Kang M, Xin H, Yuan J, et al. Transmission dynamics and epidemiological characteristics of Delta variant infections in China. medRxiv 2021; : 2021.08.12.21261991.

28 Ryu S, Kim D, Lim J-S, Ali ST, Cowling BJ. Changes in the serial interval and transmission dynamics associated with the SARS-CoV-2 Delta variant in South Korea. medRxiv 2021; : 2021.08.18.21262166.

29 Kissler SM, Fauver JR, Mack C, et al. Viral dynamics of SARS-CoV-2 variants in vaccinated and unvaccinated individuals. medRxiv 2021; : 2021.02.16.21251535. 
medRxiv preprint doi: https://doi.org/10.1101/2021.10.21.21265216; this version posted October 23, 2021. The copyright holder for this preprint (which was not certified by peer review) is the author/funder, who has granted medRxiv a license to display the preprint in perpetuity.

It is made available under a CC-BY-NC 4.0 International license .

344

$30 \mathrm{Li} \mathrm{B}$, Deng A, Li K, et al. Viral infection and transmission in a large, well-traced outbreak caused by the SARS-CoV-2 Delta variant. medRxiv 2021; : 2021.07.07.21260122.

31 Ong SWX, Chiew CJ, Ang LW, et al. Clinical and virological features of SARS-CoV-2 variants of concern: a retrospective cohort study comparing B.1.1.7 (Alpha), B.1.315 (Beta), and B.1.617.2 (Delta). Clin Infect Dis 2021; published online Aug 23. DOI:10.1093/cid/ciab721.

32 Hart WS, Maini PK, Yates CA, Thompson RN. A theoretical framework for transitioning from patient-level to population-scale epidemiological dynamics: influenza A as a case study. $J R S o c$ Interface 2020; 17: 20200230.

\section{Data sharing}

All data generated or analysed during this study are available in this published article and its supplementary files. Data and code for reproducing our results are available at https://github.com/will-s-hart/variant generation times.

\section{$\underline{\text { Acknowledgements }}$}

The data collection for the study was undertaken by the UKHSA (an executive agency of the Department of Health) as part of the COVID-19 response. WSH was funded by an EPSRC Excellence Award for his doctoral studies (Grant Reference EP/R513295/1). EM receives support from the National Institute for Health Research (NIHR) Health Protection Research Unit in Immunisation at the London School of Hygiene and Tropical Medicine in partnership with the UKHSA (Grant Reference NIHR200929). SF receives funding from the Wellcome Trust (Grant Reference 210758/Z/18/Z). RNT receives support from the UKRI (Grant Reference EP/V053507/1). Thanks to the household members who took part in this study, the nursing staff at the UKHSA who recruited and followed up the households, the laboratory staff at the UKHSA who tested the swabs and the UKHSA administrative staff who arranged for the delivery and collection of testing kits from households. The household surveillance protocol was approved by the UKHSA Research Ethics and Governance Group as part of the portfolio of the UKHSA's enhanced surveillance activities in response to the pandemic. Oral 
medRxiv preprint doi: https://doi.org/10.1101/2021.10.21.21265216; this version posted October 23, 2021. The copyright holder for this preprint

(which was not certified by peer review) is the author/funder, who has granted medRxiv a license to display the preprint in perpetuity.

It is made available under a CC-BY-NC 4.0 International license .

371 informed consent for sampling and follow up was obtained by the nurses from household

372 members who were free to decline to participate in the surveillance at any time. Consent for

373 children was obtained by a parent or legal guardian. Only anonymised data were provided to

374 non-UKHSA authors.

\section{Authors' contributions}

376 Conceptualization: WSH, SF, RNT; Literature search: WSH, RNT; Data collection and

377 curation: EM, NJA, PW; Formal analysis: WSH; Investigation: WSH; Methodology: WSH,

378 SF, RNT; Project administration: SF, RNT; Software: WSH; Supervision: PKM, SF, RNT;

379 Validation: WSH; Visualization: WSH; Writing - original draft: WSH, RNT; Writing -

380 review \& editing: all authors.

381 Declaration of interests

382 The authors declare no conflicts of interest. 